\title{
The coefficient of normal restitution for the Hertzian contact of two rough spheres colliding in a viscous fluid
}

\author{
Julian A. Simeonov \\ ${ }^{1}$ Marine Geosciences Division, Naval Research Laboratory, Stennis Space Center, MS 39529
}

\begin{abstract}
We use previous theoretical results for the added mass, history and lubrication forces between two spheres colliding in a fluid with viscosity $v$ to investigate the effect of viscous dissipation on the coefficient of restitution during contact. We assume that the mechanical interaction is governed by Hertzian mechanical contact of small duration $\tau$ and that the minimum approach distance between particles is approximately equal to the height $\sigma$ of surface micro-asperities. A non-dimensionalization of the equation of motion indicates that the contact dynamics is governed by two parameters - the ratio $\epsilon$ of the surface roughness $\sigma$ and the sphere radius $a$, and a contact Stokes number defined as $S t_{c}=\sigma^{2} / v \tau$. An asymptotic solution of the equation of motion in the limit of small $\epsilon / S t_{c}$ is used to obtain an explicit expression for the coefficient of restitution during contact and the latter is compared with estimates based on numerical solutions of the non-linear equation of motion.
\end{abstract}

Keywords: particle collisions, coefficient of restitution, Hertzian contact, lubrication force

\section{Introduction}

The coefficient of restitution $e$ is a key parameter describing the dynamics of particle collisions and the dissipation of kinetic energy in granular and particle-laden flow. While the coefficient of restitution for collisions in viscous fluids has been the subject of many investigations, most of the attention has been directed to the hydrodynamic damping during the approach of the particles and the question of viscous dissipation during mechanical contact has received little attention. Experimental investigations [1, 2, 3, 4] have found that lubrication forces generated by the drainage of the viscous fluid film in the gap of approaching spheres results in a significant damping of the sphere momentum (and a reduction of $e$ ) for collisions with low impact Stokes number $S t=\frac{m_{*} W_{\infty}}{6 \pi \rho v a_{*}^{2}}$, where $a_{*}=a_{1} a_{2} /\left(a_{1}+a_{2}\right)$ and $m_{*}=m_{1} m_{2} /\left(m_{1}+m_{2}\right)$ are the sphere reduced radius and mass, $W_{\infty}$ is the "impact" velocity (at separations where lubrication effects are negligible), and $v$ and $\rho$ are the fluid viscosity and density. The laboratory experiments of sphere-wall collisions in a viscous fluid [1, 2, 3] have focused on estimating an effective coefficient of restitu-

Email address: julian.simeonov@nrlssc.navy.mil (Julian A. Simeonov) tion $e_{w e t}$ which includes the strong hydrodynamic interaction of the particles prior to making contact. The laboratory experiments have established that $e_{w e t}$ decreases at low impact velocities and is a well-defined function of the collision Stokes number $S t$, such that $e_{w e t}$ becomes zero below a critical $S t \approx 10$. Our focus here is on the contact dynamics during viscous collisions of small particles in industrial and marine sediment transport flows where the impact Stokes numbers are typically less than 100 and where the coefficient of restitution is reduced significantly below $e=1$.

Existing estimates of $e_{\text {wet }}$ based on integrating Newton's second law for the particle motion (e.g Barnocky and Davis [1]) typically neglect the hydrodynamic forces during mechanical contact, and assume that all the viscous dissipation occurs before and after the particles make contact. For mechanical contact characterized with a coefficient of restitution $e_{d r y}$, a simple prediction

$$
e_{w e t}=e_{d r y}-\left(1+e_{d r y}\right) S t^{-1} \log \left(\sigma_{0} / \sigma\right)
$$

was obtained in Joseph et al. [3] by integrating Newton's equation for the motion of a sphere subject to lubrication force from some small initial separation $\sigma_{0}$ to a minimum separation distance corresponding to the size $\sigma$ of surface microasperities. It was found that the 
model prediction is consistent with the observed dependence of $e_{w e t}$ on $W_{\infty}$. However, we note that the comparisons of equation (1) with measurements (e.g. Joseph et al. [3], Yang and Hunt [4]) utilize $e_{d r y}<1$ which implies that additional dissipation occurs during contact. Marshall [5] extended the $e_{\text {wet }}$ model of Barnocky and Davis by including viscous damping during contact based on an approximate model for the viscous corner flow associated with the lateral expansion of the contact region. The purpose of the present paper is to investigate the effect of hydrodynamic dissipation during contact based on an asymptotic solution for the viscous gap flow [6] and to estimate the corresponding reduction of the coefficient of restitution during Hertzian contact, hereinafter denoted by $e_{c o n}$, below the theoretical value $e_{c o n}=1$. The goal of this investigation is to determine the dependence of the coefficient of restitution on the particle roughness, the impact velocity, and the material properties of the solids and the interstitial fluid.

\section{Coefficient of restitution in dry contacts}

The modeling of collisions begins with understanding the purely mechanical interaction during dry contacts where hydrodynamic forces are negligible. Extensive laboratory studies have demonstrated (see Goldsmith [7]) that the Hertzian elastic theory of contact is adequate for describing the dynamics of the mechanical contact during moderate collisions of hard spheres in air where hydrodynamic dissipation is negligible. It is well-known that the Hertzian force between two spherical particles is proportional to the 3/2-power of the overlap distance $\Delta$ at the point of contact [7]

$$
F_{H}=-k_{H} \Delta^{3 / 2},
$$

where the Hertzian stiffness parameter $k_{H}$ is related to the Young's moduli $E_{1}$ and $E_{2}$, and the Poisson ratios $\lambda_{1}$ and $\lambda_{2}$ of the two solids as follows

$$
\begin{gathered}
k_{H}=\frac{2}{3} E_{*} a_{*}^{1 / 2} ; \\
E_{*}=\frac{2}{\frac{1-\lambda_{1}^{2}}{E_{1}}+\frac{1-\lambda^{2}}{E_{2}}} ;
\end{gathered}
$$

For given impact velocity $\bar{W}$, the maximum Hertzian deformation of the particle boundaries is

$$
\delta_{H}=\bar{W} \tau_{*}
$$

$$
\tau_{*}=\left[\frac{25 m_{*}^{2}}{16 \bar{W} k_{H}^{2}}\right]^{1 / 5} .
$$

When the impact velocity is greater that a critical value (Johnson [8])

$$
v_{\text {yield }}^{2} \approx 106 a_{*}^{3} Y^{5} /\left(m_{*} E^{4}\right)
$$

related to the yield strength $Y$ of the material, most of the energy dissipation during dry collisions results from plastic deformation; for most materials, this critical velocity is on the order of a few $\mathrm{cm} / \mathrm{s}$. When the impact velocity is less than the critical value $v_{y i e l d}$, only viscoelasticity of the solid is thought to dissipate energy in dry collisions. The general form of the total mechanical force in such viscoelastic collisions is obtained by adding a dissipative force proportional to the strain rate $\dot{\Delta}$ (Kuwabara and Kono [9], Schafer et al. [10], Brilliantov et al. [11], Falcon et al. [12]) to the Hertzian force $2 \mathrm{a})$,

$$
F=-k_{H} \Delta^{3 / 2}-\mu \dot{\Delta}|\Delta|^{\gamma},
$$

where $\mu$ is a parameter related to the internal frictional dissipation in the solid.

The dissipation in such viscoelastic collisions can be analyzed by non-dimensionalizing the equation of motion for the particle pair

$$
m_{*} \frac{d W}{d t}=-k_{H} \Delta^{3 / 2}-\mu \dot{\Delta}|\Delta|^{\gamma}
$$

using the initial impact velocity $\bar{W}$ and the Hertzian time scale to define the non dimensional velocity $w=W / \bar{W}$, non-dimensional time $\tau=t / \tau_{*}$ and non-dimensional displacement $\delta=\Delta /\left(\tau_{*} \bar{W}\right)$. It is then found that the resulting non-dimensional equation

$$
\frac{d w}{d \tau}=-\frac{5}{4} \delta^{3 / 2}-\frac{\mu \tau_{*}\left(\bar{W} \tau_{*}\right)^{\gamma}}{m_{*}} \dot{\delta}|\delta|^{\gamma},
$$

depends on a single dimensionless parameter $\mu \tau_{*} m_{*}^{-1}\left(\bar{W} \tau_{*}\right)^{\gamma}$ that represents the ratio of the restoring and dissipative mechanical forces. Multiplying by $w=\dot{\delta}$ and integrating the result during the contact period in the limit of weak dissipation $\mu \rightarrow 0$, it is possible to obtain an analytical expression for the coefficient of restitution (see Kuwabara and Kono [9], Falcon et al. [12], also Ray et al. [13])

$$
e_{d r y}=1-\frac{4}{5} \mathcal{B}\left(\frac{3}{2}, \frac{2(\gamma+1)}{5}\right) \frac{\mu \tau_{*}}{m_{*}}\left(\tau_{*} \bar{W}\right)^{\gamma},
$$


where $\mathcal{B}$ is the Beta function. The case $\gamma=1 / 2$ corresponds to a viscoelastic solid and predicts a dry coefficient of restitution that decreases weakly with the impact velocity proportional to $-\bar{W}^{1 / 5}$. Verification of the prediction (9) for $\gamma=1 / 2$ has been difficult due to lack of measurement data for $\mu$ which is related to the solid viscosity coefficients.

For rough surfaces, the range of plastic dissipation extends to much lower velocity as the particle radius in the yield condition (5) is replaced by the effective asperity curvature radius. The Hertzian force model (2a) is valid for rough surfaces only at high loads. At low loads, the presence of surface roughness tends to increase the effective contact area (relative to Hertzian contact of smooth surfaces) and leads to an approximately linear elastic force [20]. The detailed investigation of such complex multi-asperity contact models (see [21] for a recent review) is beyond the scope of the present paper where the focus is on the viscous dissipation due to the interstitial fluid. We will illustrate the effect of viscous dissipation using the Hertzian contact model which is commonly assumed to be valid over the entire range of loads in numerical models of particleladen flows. The investigation of slow viscous collisions in the following section will show that hydrodynamic effects can give rise to a dissipative term similar to the second term in equation (6).

\section{Coefficient of restitution in viscous contacts}

The main obstacle to evaluating the effect of hydrodynamic forces during contact has been the lack of theoretical model or measurements of these forces. For that reason, numerical modelers (e.g. Derksen and Sundaresan [14], Simeonov and Calantoni [15]) have used various ad hoc assumptions to limit the magnitude of the lubrication force during contact. Here, we should point that classic theories for the lubrication force (e.g. Cooley and O'Neill [16]) are based on the steady Stokes equation and, as such, are not appropriate to describe the hydrodynamics during mechanical contact when the particle velocity changes very rapidly and imparts large accelerations on the surrounding fluid.

The unsteady hydrodynamics of colliding particles was recently investigated in [6], where following Barnocky and Davis [1], the particles were assumed to make contact through microasperities of height $\sigma$ so that the gap between the particles remains finite during contact. This asperity contact model should be con- 122 trasted with the elastohydrodynamic theory of Davis ${ }_{123}$ et al. [25] for the viscous collision of smooth parti- ${ }_{124}$ cles where the minimum separation between the particles $\delta_{E H L}$ becomes so small that the fluid pressure in the gap generated by the squeezed flow becomes sufficiently large to transmit the mechanical stresses without actual contact. For low impact collisions, the minimum approach distance $\delta_{E H L}$ is typically much smaller than real particle roughness which is in the range of 0.01-1 $\mu m$ [3]. For example, using the estimate of Yang and Hunt [26]

$$
\delta_{E H L}=\frac{a}{3}\left(\frac{4\left(1-\lambda^{2}\right)}{\pi \sqrt{2}}\right)^{2 / 5}\left(\frac{\nu \bar{W}}{a c^{2}}\right)^{2 / 5},
$$

where $c=\sqrt{E_{*} / \rho_{s}}$ is the one-dimensional sound speed, we find $\delta_{E H L}=1.410^{-6} \mathrm{~mm}$ for two $1 \mathrm{~mm}$ quartz spheres $(c=5000 \mathrm{~m} / \mathrm{s})$ colliding in water with impact velocity $\bar{W}=1 \mathrm{~mm} / \mathrm{s}$. Therefore, boundary deformations due to elastohydrodynamic effects are ignored in our collision model focusing on low impact collisions.

Analytical progress in estimating the lubrication forces during the collision of rough spheres is only possible if we ignore the deviation from spherical geometry introduced by the asperities and its effect on the gap flow. In reality, the presence of asperities will further restrict the fluid flow in the gap leading to an increase of the lubrication forces. Thus, the presented analytical model would provide a lower bound estimate of the viscous dissipation during collision of rough spheres. To determine the unsteady hydrodynamic forces during contact, we solved the time-dependent Stokes equations for the viscous flow in the vicinity of two slowly colliding non-rotating spheres whose diameter ratio is $k$ [6]. The initial value problem was solved using Laplace transform and an initial condition corresponding to the steady state solution of Cooley and O'Neill [16] for the approach of two spheres in a viscous fluid.The solution was obtained using a singular perturbation expansion in the small parameter $\epsilon=\sigma / a$ and the Stokes streamfunction approach of Cooley and O'Neill [16]. Tangent-sphere coordinates were used to evaluate the viscous solution outside the gap between the particles. The asymptotic theory was limited to the interaction of relatively smooth particles such that the viscous time scale in the gap $\sigma^{2} / v$ is of the same order or smaller than the Hertzian contact time scale $\tau_{*}$. It will be shown below that the ratio of these two time scales, which we refer to as the contact Stokes number

$$
S t_{c}=\frac{\sigma^{2}}{v \tau_{*}},
$$

describes the relative importance of the mechanical forces and the viscous dissipation. Below, we investigate the dependence of the coefficient of restitution on 
the particle roughness $\sigma$, the material stiffness repre- ${ }_{147}$ sented by $\tau_{*}$ or $S t_{c}$, the diameter ratio $\kappa$ and the impact ${ }_{148}$ velocity $\bar{W}$.

In the related paper mentioned above [6], we obtained ${ }_{150}$ the following result for the time-dependent hydrody- 15 namic force during the collisional contact of a sphere of radius $a$ with a fixed motionless sphere of radius $a / \kappa$,

$$
\begin{aligned}
& f=-\frac{4}{3} \pi \rho a^{3} C_{M} \frac{d W}{d t}-3 \pi \rho \sqrt{v} a^{2} K \int_{0}^{t} \frac{d W}{d \tau} \frac{d \tau}{\sqrt{\pi(t-\tau)}} \\
& -\frac{6 \pi \rho v a^{2} W}{(1+\kappa)^{2} \sigma},
\end{aligned}
$$

where $C_{M}=3 K / 4-1$ is the added mass coefficient,

$$
K(\kappa)=\int_{0}^{\infty} \frac{\lambda^{2} e^{-\lambda}}{1-e^{-\lambda(1+\kappa)}} d \lambda
$$

is an $\mathrm{O}(1)$ function of $\kappa$ and the first, second and third 156 term in 12 correspond to the added mass, history and ${ }^{157}$ lubrication forces, respectively. In the two limits $K=0$ (wall) and $\kappa \rightarrow \infty$ (single particle), our estimates for 159 the added mass coefficient $C_{M}=3 \zeta(3) / 2-1 \approx 0.803{ }^{160}$ and $C_{M}=1 / 2$ were found to agree with previous cal- ${ }^{161}$ culations [18, 19, 22, 23, 24]. We should note that 162 the last term in (12) has the same form as the classi- ${ }^{163}$ cal leading order result [16] except that the gap sep- ${ }^{164}$ aration is replaced by the fixed height of the asperity. This is not necessarily a contradiction because during the Hertzian compression the gap decreases from $\sigma$ to $\sigma-\delta_{H}$ where $\delta_{H}=\bar{W} \tau_{*}=\sigma \epsilon \operatorname{Re} / S t_{c}$ is much smaller than $\sigma$ for the considered low Reynolds number collisions $R e=a \bar{W} / v<<1$ provided that $S t_{c}>\epsilon$; the small $O\left(\epsilon \operatorname{Re} / S t_{c}\right)$ term, as well as other higher order terms, are therefore not included in the leading order force estimate $(12)$.

\subsection{Asymptotic estimate for small $\epsilon / S t_{c}$}

Using the theoretical estimate in (12), we can now analyze the effect of the hydrodynamic forces on the collision process based on Newton's equation for the particle motion

$$
\begin{aligned}
& m_{K} \frac{d W}{d t}=-3 \pi \rho \sqrt{v} a^{2} K \int_{0}^{t} \frac{d W}{d \tau} \frac{d \tau}{\sqrt{\pi(t-\tau)}} \\
& -\frac{6 \pi \rho v a^{2} W}{(1+\kappa)^{2} \sigma}-k_{H} \Delta^{3 / 2},
\end{aligned}
$$

where the added mass force in $(12)$ has been incorporated into the left hand side via the effective mass parameter

$$
m_{K}=\frac{4}{3} \pi \rho_{s} a^{3}\left[1+C_{M} \frac{\rho}{\rho_{s}}\right]
$$

The initial conditions correspond to the onset of contact when $W(t=0)=\bar{W}$ and $\delta(t=0)=0$.

We can again identify the pertinent non-dimensional parameters by non-dimensionlazing the equation of motion using the initial impact velocity $|\bar{W}|$ and the modified time scale $\tau_{K}=\left(\frac{25 m_{K}^{2}}{16 k_{H}^{2} \bar{W}}\right)^{1 / 5}$ to define the non dimensional velocity $w=W / \bar{W}$, non-dimensional time $\tau=$ $t / \tau_{K}$ and non-dimensional displacement $\delta=\Delta /\left(\tau_{K} \bar{W}\right)$. The resulting non-dimensional equation

$$
\begin{aligned}
& \frac{d w}{d \tau}=-3 K\left(1+\rho C_{M} / \rho_{s}\right)^{-4 / 5} \frac{3 \rho \epsilon}{4 \rho_{s} S t_{c}^{1 / 2}} \int_{0}^{\tau} \frac{d w}{d \tilde{\tau}} \frac{d \tilde{\tau}}{\sqrt{\pi(\tau-\tilde{\tau})}} \\
& -\left(1+\rho C_{M} / \rho_{s}\right)^{-3 / 5} \frac{3 \rho \epsilon}{4 \rho_{s} S t_{c}} \frac{6 w}{(1+\kappa)^{2}}-\frac{5}{4} \delta^{3 / 2},
\end{aligned}
$$

indicates that the two non-dimensional parameters $\epsilon / S t_{c}^{1 / 2}$ and $\epsilon / S t_{c}$ describe the relative magnitude of the history and resistance lubrication force with respect to the $O(1)$ Hertzian force. For small contact Stokes numbers $S t_{c}<<1$, the history term is $O\left(S t_{c}^{1 / 2}\right)$ smaller than the lubrication force term and the leading order $O\left(\epsilon / S t_{c}\right)$ of equation (16) corresponds to equation (8) with $\gamma=0$ and the coefficient $\mu \tau_{*} / m_{*}$ replaced by $\left(1+\rho C_{M} / \rho_{S}\right)^{-3 / 5} \frac{3 \rho \epsilon}{4 \rho_{s} S t_{c}} \frac{6}{(1+\kappa)^{2}}$. The respective asymptotic estimate of the coefficient of restitution for small $\epsilon / S t_{c}$ becomes

$e_{c o n}=1-\frac{4}{5} \mathcal{B}\left(\frac{3}{2}, \frac{2}{5}\right)\left(1+\rho C_{M} / \rho_{s}\right)^{-3 / 5} \frac{3 \rho \epsilon}{4 \rho_{s} S t_{c}} \frac{6}{(1+\kappa)^{2}}$.

We first note that the above estimate of the coefficient of restitution depends weakly on the added mass coefficient $C_{M}$ because the exponent and the factor $\rho / \rho_{s}$ are less than one. Equation (17) also indicates that the effect of viscous damping decreases as the diameter of the target particle decreases ( $\kappa$ increases). The case with the largest reduction of $e_{c o n}$ corresponds to a collision with a wall $(\kappa=0)$ and will be the focus of the remaining discussion. Our analytical estimate (17) finally shows that $1-e_{c o n}$ is inversely proportional to the parameter $S t_{c} / \epsilon$. Assuming $\kappa=0$ and using equations $1142 \mathrm{~b}$, we can obtain the following estimate

$$
\frac{S t_{c}}{\epsilon}=\left(\frac{\bar{W}}{4 \pi^{2} c}\right)^{1 / 5} \frac{c \sigma}{v} .
$$
does not depend on the particle radius $a$ and that the decrease of $e_{c o n}$ from 1.0 is inversely proportional to the 
surface roughness $\sigma$ and the one-fifth power of the impact velocity.

To discuss the relevant range of the parameter $S t_{c} / \epsilon$, we next consider the specific case of quartz particles with $\lambda=0.1, \rho_{s}=2.6 \rho$ and $c=5000 \mathrm{~m} / \mathrm{s}$ colliding in water with initial impact velocity $\bar{W}=1 \mathrm{~mm} / \mathrm{s}$. Then, for typical surface roughness $0.01 \mu m<\sigma<1 \mu m$ of particles used in laboratory experiments [3], we have the following range $1<S t_{c} / \epsilon<100$. Noting the weak dependence on $\bar{W}$ (e.g. a 100-fold increase in $\bar{W}$ results in a factor of 2.5 increase in $S t_{c}$ ) below we will use the range $1<S t_{c} / \epsilon<100$ to investigate the dependence of $e_{c o n}$ on $S t_{c} / \epsilon$.

\subsection{Numerical estimate for arbitrary $\epsilon / S t_{c}$}

Next, we will compare the asymptotic estimate in (17) with the numerical integration of the full equation of motion (16). We integrate the equation of motion (16) and the corresponding kinematic condition $d \delta / d t=w$ using a simple forward Euler scheme

$$
w_{n+1}=w_{n}+\dot{w}_{n} \Delta t \quad \delta_{n+1}=\delta_{n}+w_{n} \Delta t,
$$

where $\Delta t$ is the time step and the rate of change of velocity $\dot{w}_{n}$ at time $t_{n}$ is estimated from (16). The main complication in the numerical treatment is the history integral which makes equation (16) an implicit integrodifferential equation for the acceleration $\dot{w}$. It is however possible to evaluate the history integral at time $t_{n}$ in terms of data at previous times $\left(\dot{w}_{0}, \ldots, \dot{w}_{n-1}\right)$, by splitting the history integral in two intervals $\left(0, t_{n-1}\right)$ and $\left(t_{n-1}, t_{n}\right)$, using integration by parts in the second interval and evaluating the result with quadratures. For more details and a discussion of the numerical accuracy of the scheme, the reader is referred to Simeonov [6] where it was demonstrated that the numerical error in the predicted coefficient of restitution was less than $0.3 \%$ when the non-dimensional time step was $\Delta t=0.001$. The same time step was used for the simulations presented ${ }_{228}$ below.

By integrating numerically equation subject to 229 the initial condition $w=-1$ and $\delta=0$, we were able 230 to estimate the coefficient of restitution $e_{c o n}$ which is es- ${ }_{231}$ sentially the value of $w$ at the end of contact when $\delta=0 \quad 232$ again. For the collision of a quartz sphere with a quartz ${ }_{233}$ wall for the following range $1<S t_{c} / \epsilon<100$ of the ${ }_{234}$ main non-dimensional parameter and for two different 235 values of $\epsilon$. The numerically computed $e_{\text {con }}$ is shown ${ }_{236}$ as a function of $\epsilon / S t_{c}$ in Figure 1 (symbols) along with ${ }_{237}$ the analytical estimate (straight line). Figure 1 shows 238 that there is a significant reduction of $e_{c o n}$ as the param- 239 eter $\epsilon / S t_{c}$ approaches $\mathrm{O}(1)$. As expected, the numer- 240
Figure 1: The coefficient of restitution during contact as a function of the non-dimensional parameter $S t_{c} / \epsilon$ for the normal collision of a quartz sphere with a quartz wall $(\kappa=0)$. The symbols correspond to the numerically computed coefficient of restitution for two different values of $\epsilon$ and the straight line corresponds to the analytical solution in equation 17 .

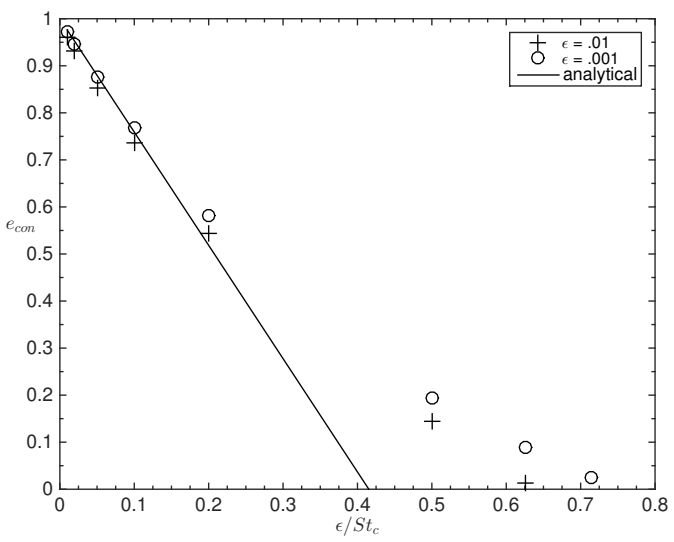

ical solution is adequately represented by the asymptotic result at small values of $\epsilon / S t_{c}$. The analytical solution tends to overestimate the reduction of $e_{c o n}$ only at very large $\epsilon / S t_{c}>0.2$ corresponding to very smooth (or soft) particles. Therefore, for most practical applications involving hard particles, the analytical estimate of the contact coefficient of restitution should be adequate in describing the viscous dissipation. We should mention that the significant deviation of $e_{\text {con }}$ (Figure 1 , symbols) at large $\epsilon / S t_{c}$ from the linear dependence is not due to the $O\left(\epsilon / S t_{c}^{1 / 2}\right)$ history forces, but is rather due to higher order $O\left(\epsilon / S t_{c}\right)$ terms in the asymptotic expansion of the non-linear Hertzian force in $(16)$. For example, suppressing the history force in the simulation for $\epsilon / S t_{c}=0.5$ only increases $e_{c o n}$ by $10 \%$.

\section{Discussion}

It may be instructive to use our theory to estimate the effect of viscous dissipation on the contact coefficient of restitution in laboratory experiments of sphere-wall collisions at low Stokes numbers. To make such estimates, we used the experimental conditions from the laboratory experiments of Joseph et al. [3] for the low St collision of nylon, glass and steel spheres with a zerodur wall (Table 1).

Using the data in Table 1, we computed $\epsilon / S t_{c}$ and estimated $e_{c o n}$ from equation (17). The resulting $e_{c o n}$ given in Table 1 is substantially lower than the measured (Joseph et al. [3]) $e_{d r y}=0.97$ for the relatively 
smooth glass and steel spheres with $d=4.1 \mathrm{~mm}$ and 293 $d=6.35 \mathrm{~mm}$. Our estimates imply that viscous dis- 294 sipation during contact may be important even for the ${ }^{295}$ relatively large laboratory particles when their rough- ${ }_{297}^{296}$ ness does not exceed $O(0.1 \mu \mathrm{m})$. We note that the values 298 of $e_{c o n}$ given in Table 1 are based on $W_{\infty}$, which may ${ }^{299}$ significantly overestimate the impact velocity $\bar{W}$ due to ${ }_{301}^{300}$ viscous dissipation prior to impact. Therefore, the val- ${ }_{302}$ ues of $e_{\text {con }}$ given in Table 1 would slightly underestimate ${ }_{303}$ the damping by viscous dissipation.

The coefficient of restitution during contact $e_{\text {con }}$ is ${ }^{305}$ usually taken to be constant and equal to the coefficient ${ }_{307}$ of restitution in dry collisions, $e_{d r y}$. Here, we have de- 308 veloped a theoretical model for collisions in a viscous ${ }^{309}$ fluid, which suggests that the coefficient of restitution ${ }_{311}^{310}$ during contact $e_{c o n}$ depends on two non-dimensional ${ }_{312}$ parameters - the non-dimensional particle roughness ${ }^{313}$ $\epsilon=\sigma / a$ and $S t_{c} / \epsilon$. In the limit of small $\epsilon / S t_{c}$ and ${ }^{314}$ small $S t_{c}$, an asymptotic expression shows that the de- ${ }_{316}$ crease of $e_{c o n}$ from 1.0 is inversely proportional to the ${ }_{317}$ surface roughness and the one-fifth power of the particle ${ }^{318}$ impact velocity. It was found that $e_{c o n}$ is significantly ${ }_{320}^{319}$ reduced below $e_{c o n}=1$ as the contact Stokes number ${ }_{321}{ }^{320}$ $S t_{c}$ approaches the non-dimensional particle roughness 322 $\epsilon$. The asymptotic prediction for $e_{\text {con }}$ was validated with ${ }^{323}$ numerical simulations that retained the fully non-linear ${ }_{325}{ }_{324}$ aspect of Hertzian contact. The obtained theoretical ex- ${ }_{326}$ pression for $e_{c o n}$ can be used to model the collision of ${ }^{327}$ particles in numerical simulations that do not resolve ${ }^{328}$ explicitly the thin layer lubrication effects during colli- ${ }_{330}^{329}$ sions in a viscous fluid [27, 28, 29, 30, 31].

\section{Acknowledgment}

This work was supported by the Office of Naval Research through base funding to the Naval Research Laboratory.

[1] G. Barnocky and R.H. Davis, "Elastohydrodynamic collision and rebound of spheres: Experimental verification," Phys. Fluids 31 (6), 1324-1329 (1988).

[2] P. Gondret, E. Hallouin, M. Lance and L. Petit, "Experiments on the motion of a solid sphere toward a wall: From viscous dissipation to elastohydrodynamic bouncing," Phys. Fluids 11 (9), 28032805 (1999).

[3] G. G. Joseph, R. Zenit, M.L. Hunt and A.M. Rosenwinkel, "Particle-wall collisions in a viscous fluid," J. Fluid Mech. 433, 329-346 (2001).

[4] F. L. Yang and M. L. Hunt, "Dynamics of particle-particle collisions in a viscous liquid," Phys. Fluids 18, 121506 (2006).

[5] J. S. Marshall, "Viscous damping force during head-on collision of two spherical particles," Phys. Fluids 23, 013305 (2011).

[6] J.A. Simeonov, "The unsteady hydrodynamic force during the collision of two spheres in a viscous fluid," Acta Mechanica (on- ${ }^{356}$ line) http://link.springer.com/article/10.1007/s00707-015-1465-5
[7] W. Goldsmith, Impact: the theory and physical behavior of colliding solids (Dover Publications, New York, 1960)

[8] K. L. Johnson, Contact Mechanics (Cambridge University Press, Cambridge, 1985)

[9] G. Kuwabara and K. Kono, "Restitution coefficient in a collision between two spheres," Jpn. J. Appl. Phys., 26, 1230-1233 (1987).

[10] J. Shafer, S. Dippel, and D. E. Wolf, "Force schemes in simulations of granular materials," J. Phys. I France, 6, 5-20 (1996).

[11] N. V. Brilliantov, F. Spahn, J.-M. Hertzsch, and T. Poschel "Model for collisions in granular gases," Phys. Rev. E 53, 53825392 (1996).

[12] E. Falcon, C. Laroche, S. Fauve, and C. Coste, " Behavior of one inelastic ball bouncing repeatedly off the ground," Eur. Phys. J. B, 3, 45-57 (1998).

[13] S. Ray, T. Kempe, and J. Frohlich, " Efficient modeling of particle collisions using a non-linear viscoelastic contact force," Int. J. Multiphase Flow, 76, 101-110 (2015).

[14] J. J. Derksen and S. Sundaresan, "Direct Numerical simulations of dense suspensions: wave instabilities in liquid fluidized beds," J. Fluid Mech. 587, 303-336 (2007).

[15] J. A. Simeonov and J. Calantoni, "Modeling mechanical contact and lubrication in Direct Numerical Simulations of colliding particles," Int. J. of Multiphase Flow 46, 38-53 (2012).

[16] M.D. A. Cooley and M. E. $\mathrm{O}^{\prime}$ Neill, "On the slow motion generated in a viscous fluid by the approach of a sphere to a plane wall or stationary sphere," Mathematika 16, 37-49 (1969).

[17] J.R. Smart and D. T. Leighton, "Measurement of the hydrodynamic surface roughness of noncolloidal spheres," Phys. Fluids $\mathbf{1}$, 52-60 (1989)

[18] D. Weihs and R.D. Small, "An exact solution of the motion of two adjacent spheres in axisymmetric potential flow," Israel $J$. Tech. 13, 1-6 (1975).

[19] M. Bentwich and T. Miloh, "On the exact solution for the twosphere problem in axisymmetrical potential flow" J. Appl. Mech. 45, 463-468 (1978).

[20] J. A. Greenwood and J. H. Tripp, "The elastic contact of rough spheres" J. Appl. Mech. 34, 153-159 (1967).

[21] G.G. Adams and M. Nosonovsky, "Contact modeling - forces" Tribology International 33, 431-442 (2000).

[22] D. J. Jeffrey and H.-S. Chen, "The virtual mass of a sphere moving toward a plane wall," J. Appl. Mech. 44, 166-167 (1977).

[23] A. A. Kharlamov, Z. Chara and P. Vlasak, "Hydraulic formulae for the added masses of an impermeable sphere moving near a plane wall" J. Eng. Math 62, 161-172 (2007).

[24] L. D. Landau and E.M. Lifshitz EM Fluid Mechanics (1988).

[25] R. H. Davis, J.-M. Serayssol and E.J. Hinch, "The elastohydrodynamic collision of two spheres," J. Fluid Mech. 163, 479-497 (1986).

[26] F. L. Yang and M. L. Hunt, "A mixed contact model for an immersed collision between two solid surfaces," Phil. Trans. R. Soc. A 366, 2205-2218 (2008).

[27] T. Kempe and J. Frohlich, "Collision modelling for the interface-resolved simulation of spherical particles in viscous fluids," J. Fluid Mech. 709, 445-489 (2012).

[28] A. G. Kidanemariam and M. Uhlmann, "Interface-resolved direct numerical simulation of the erosion of a sediment bed sheared by laminar channel flow," Int. J. of Multiphase Flow 67, 174-188 (2014).

[29] C. Ji, A. Munjiza, E. Avital, D. Xu and J. Williams, "Saltation of particles in turbulent channel flow," Phys. Rev. E 89, 052202 (2014).

[30] A. A. Zaidi, T. Tsuji and T. Tanaka, "Direct numerical simulation of finite sized particles settling for high Reynolds number and dilute suspension," Int. J. Heat and Fluid Flow 50, 330-341 (2014). 
[31] J. R. Finn, S. V. Apte and M. Li, " Numerical Simulations of Sand Ripple Evolution in Oscillatory Boundary Layers," ASME Fluids Engineering Division Summer Meeting 2014 1B, FEDSM2014-22065 (2014) 
Table 1: The coefficient of restitution during viscous contact $e_{c o n}$ 17) in several low Stokes number collisions of glass, nylon and steel spheres with zerodur wall in water-glycerol solutions (Joseph et al. [3]) Also given is the range of the observed wet coefficient of restitution $e_{w e t}$

\begin{tabular}{cccccccccccccc}
\hline $\begin{array}{c}d \\
{[\mathrm{~mm}]}\end{array}$ & $\begin{array}{c}E_{1} \\
{[\mathrm{GPa}]}\end{array}$ & $\lambda_{1}$ & $\begin{array}{c}\rho_{p} \\
{\left[\frac{\mathrm{kg}}{\mathrm{m}^{3}}\right]}\end{array}$ & $\begin{array}{c}\sigma \\
{[\mu \mathrm{m}]}\end{array}$ & $\begin{array}{c}E_{2} \\
{[\mathrm{GPa}]}\end{array}$ & $\begin{array}{c}\lambda_{2} \\
{\left[\frac{\mathrm{mm}^{2}}{\mathrm{~s}}\right]}\end{array}$ & $\begin{array}{c}W_{\infty} \\
{\left[\frac{\mathrm{mm}}{\mathrm{s}}\right]}\end{array}$ & $S t$ & $\epsilon \times 10^{5}$ & $\frac{\text { Stc }}{\epsilon}$ & $e_{\text {wet }}$ & $e_{\text {con }}$ \\
\hline 6.35 & 65 & 0.23 & 2540 & 0.13 & 91 & 0.24 & 5 & 50 & 17.9 & 4.1 & 34.2 & $0.15-0.3$ & 0.928 \\
\hline 4.1 & 65 & 0.23 & 2540 & 0.05 & 91 & 0.24 & 1 & 50 & 57.9 & 2.4 & 13.2 & $0.4-0.7$ & 0.813 \\
\hline 3.0 & 65 & 0.23 & 2540 & 0.138 & 91 & 0.24 & 1 & 25 & 21.2 & 9.2 & 31.6 & $0.2-0.5$ & 0.922 \\
\hline 6.35 & 2.76 & 0.4 & 1140 & 2.0 & 91 & 0.24 & 1 & 20 & 16.1 & 63 & 219 & $0.15-0.3$ & 0.979 \\
\hline 6.35 & 190 & 0.27 & 7780 & 0.0236 & 91 & 0.24 & 11.6 & 40 & 19 & 0.74 & 8.35 & 0.2 & 0.894 \\
\hline
\end{tabular}

